\title{
QUANTIFYING THE SLOPE DEFORMATION OF A LASER POINT CLOUD ACQUIRED USING MOBILE MAPPING SYSTEM
}

\author{
* Naoto Samori ${ }^{1}$, Satoshi Nishiyama ${ }^{1}$ and Koki Sakita ${ }^{1}$ \\ ${ }^{1}$ Graduate School of Environmental and Life Science, Okayama University, Japan \\ *Corresponding Author, Received: 11 Nov. 2021, Revised: 01 Dec. 2021, Accepted: 18 Dec. 2021
}

\begin{abstract}
In recent years, the increasing frequency of heavy short-duration rainfall has escalated the risk of slope collapse. Inspecting the slope to detect any deformation is crucial for preventing slope collapse. However, conventionally, these inspections are conducted using close visual inspection. As a result, the findings are primarily dependent on the experience and skill level of the inspection engineers. In this study, we used a mobile mapping system (MMS) that could efficiently acquire laser point clouds over a wide area and quantitatively evaluate the shape of the structures. Thereafter, the point cloud was used to quantify the slope deformation by applying the iterative closest point (ICP) algorithm. The effectiveness of this method in aiding slope inspection was evaluated by examining the direction and amount of slope deformation on two separate days. Thereafter, the obtained deformations were clarified by filtering them to remove point clouds, such as vegetation and vehicles. Based on the study findings, we expect the proposed method to play an important role in assisting slope inspection.
\end{abstract}

Keywords: Slope, Inspection, Point cloud, Mobile mapping system, Iterative closest point

\section{INTRODUCTION}

The increasing frequency of heavy and shortduration rainfall has amplified the possibility of slope collapse [1] that can cause enormous economic losses. For example, the collapse of a road slope associated with a transportation infrastructure can interrupt its network. To prevent the occurrence of such situations, it is important to inspect the slope and identify any deformations that indicate the possibility of collapse. Currently, slope inspections in Japan are primarily conducted through close visual inspection that involve approaching the object and observing the presence and degree of deformation [2]. Due to this, the results obtained by this method are qualitative and predominantly depend on the experience and skill of the engineers. However, since these results vary depending on the engineer and their inspection, obtaining uniform results is challenging.

In recent years, surveys utilizing laser scanners that can acquire laser point clouds have become widespread [3]; a point cloud is a collection of three-dimensional coordinates (X, Y, Z) and color information (RGB) acquired from the camera image data. A point cloud can be rotated, zoomed in and out, and allows the removal of only necessary parts. Since point clouds are expressed in coordinates, structural shapes such as slopes can be expressed quantitatively (Fig.1). A fixed-type typical laser scanner can acquire a point cloud of the surrounding area of the installation point. However, measuring slopes constructed over a wide area requires changing the installation point several times. In this study, we used a vehicle-mounted sensing device (mobile mapping system, hereinafter referred to as MMS) to effectively acquire multiple point clouds over a wide area. MMS is a sensing device mounted with a laser scanner that can be driven on the road to acquire a point cloud while simultaneously obtaining the location information and the position orientation of the vehicle (Fig.2). During the experiment, we acquired two point clouds at different times using the MMS measurements. Thereafter, we attempted to extract the deformation in the two measurements by applying the iterative closest point (ICP) algorithm.

We verified four performance parameters to test the MMS: (i) accuracy of the MMS point cloud acquisition, (ii) accuracy of the MMS point cloud reproduction, (iii) slope deformation extraction using the ICP algorithm, and (iv) extracting the deformation of the slope from a wide area based on the MMS measurements and ICP algorithm in the above three verifications. Thereafter, based on the obtained results, we considered whether the proposed method aids accurate slope inspection.

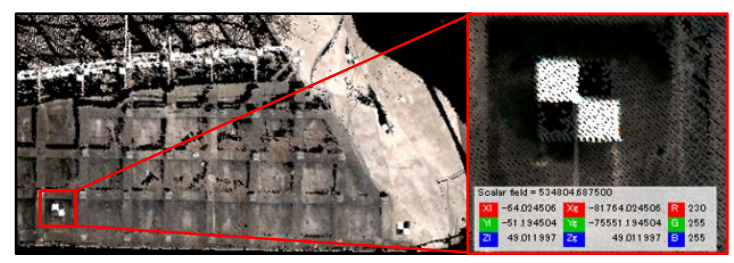

Fig.1 Laser point cloud of the slope. The numbers present the coordinates of the center of the clipped target point cloud 


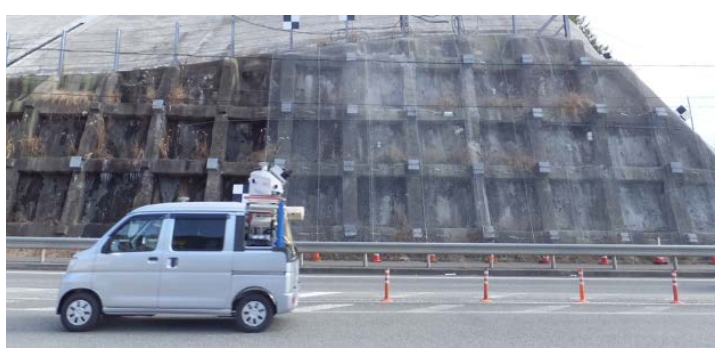

Fig. 2 The MMS vehicle acquiring the point clouds of its surroundings

\section{RESEARCH OUTLINE}

This chapter describes the proposed MMS, the ICP algorithm used in this study, the slope of the target, and its measurement summary.

\subsection{Mobile Mapping System (MMS)}

MMS is a sensing device that can continuously acquire a laser point cloud (Fig.3). The MMS system can be broadly divided into two parts: a global navigation satellite system (GNSS)/inertial measurement unit (IMU)/odometry combined navigation system for measuring the vehicle position and attitude, and a system for acquiring information around the vehicle. The former part consists of a GNSS, an IMU, and an odometer, and the latter part consists of a laser scanner and a camera. MMS can acquire accurate 3D coordinates around roads [4]. At present, it is expected to be utilized for creating 3D topographic maps for road facilities [5], managing river embankments [6], and surveying civil engineering structures [7].

\subsection{Iterative Closest Point (ICP) Algorithm}

The ICP algorithm [8] is considered to be capable of extracting deformations from point clouds obtained on two separate days. ICP is a scanmatching algorithm known as simultaneous localization and mapping that performs simultaneous self-localization and mapconstruction (Fig.4); scan-matching is a technique for aligning two point clouds to match their shapes. The working principle of the ICP algorithm is as follows. First, the algorithm requires the initial positions of the two point clouds for a rough alignment. Thereafter, the point cloud is divided into a square grid called a mesh (Fig.5). Next, the algorithm maps the points of the two separate days on the points of the same position in the mesh. Then, it optimizes the position of the entire point cloud (rotation and translation) to obtain the least value of sum of the distances between the mapped points. This set of mapping and optimization steps is repeated for a specified number of iterations and then terminated. Lastly, the coordinate values of the points that are associated with the two point clouds are compared. The algorithm uses the mean value of the vectors in each mesh; this value is considered to be the amount of change in the mesh. Based on the above, it is considered that ICP can express the change in the two point clouds as a vector.

For this study, we set the number of ICP iterations for verification as 15 .

\subsection{Target Slope and Its Measurement Summary}

The slope considered for this study had an approximate length and height of $70 \mathrm{~m}$ and $10 \mathrm{~m}$, respectively (Fig.6). A total of 14 targets were set up on the slope for verification, each with a size of $0.7 \times 0.7 \mathrm{~m}^{2}$ on one side. Verification (i) utilized

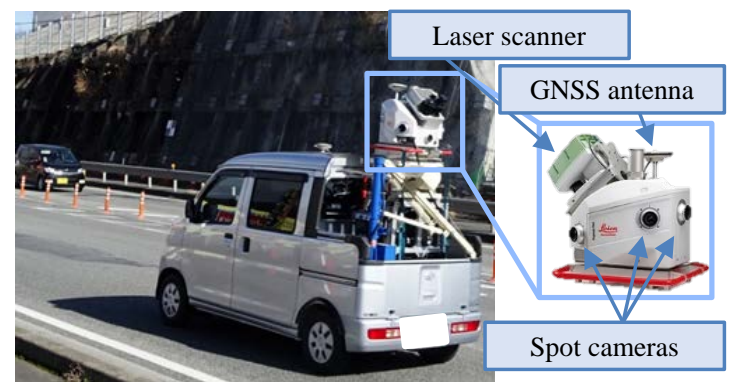

Fig.3 MMS equipment mounted on a vehicle

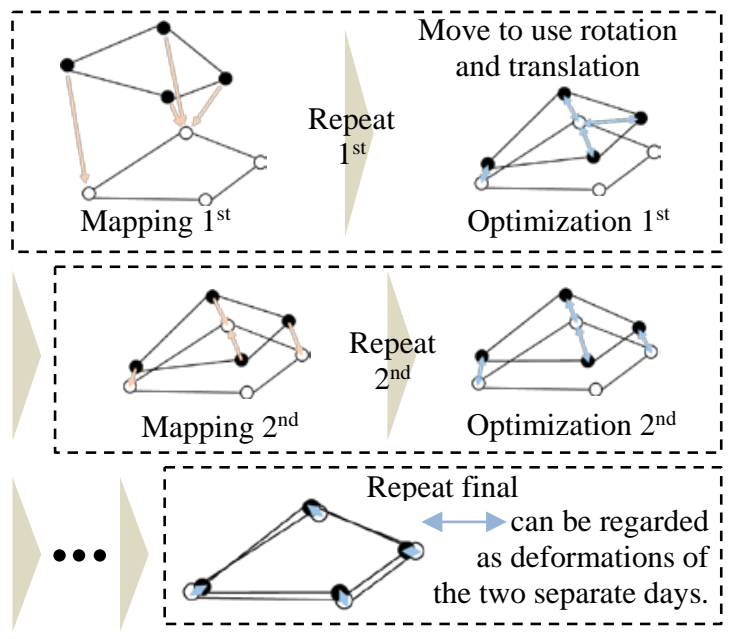

Fig.4 Mechanism for calculating the amount of deformation using ICP

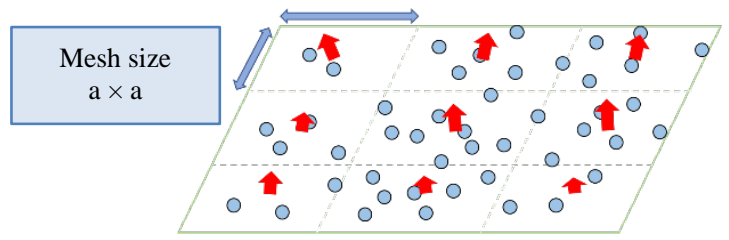

Fig.5 Mesh size for applying the ICP algorithm 


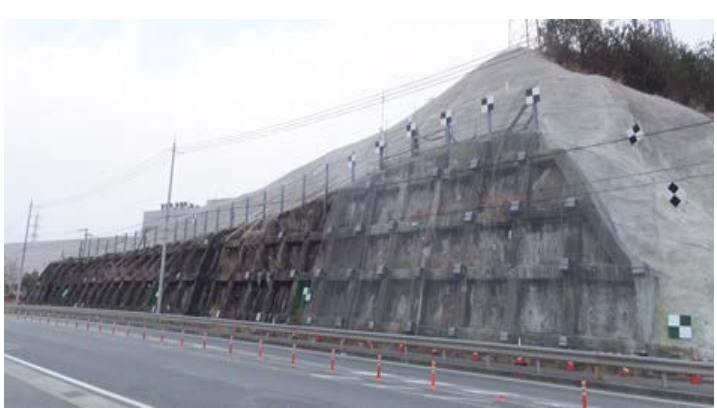

Fig.6 The slope of the research object

these targets whereas Verifications (ii), (iii), and (iv) utilized their respective ranges (Fig.7). Eight of these targets were set as the reference points (TG) for adjustment, and the remaining six were set as the verification points (KS); adjustment reference points are used to correct the misalignment of the point cloud acquired by the MMS measurement. For adjustment, we used the coordinates of the reference points in the slope acquired by the MMS measurement and the coordinates of the reference points acquired by the total station (TS). The verification points were utilized to evaluate the accuracy of the acquired point cloud in Section 3.

The MMS and TS measurements techniques are as follows. For the MMS measurement, we used the Leica Geosystems mobile mapping solution "Pegasus: Two" [9]; this measurement system is a non-transparent phase-contrast type system with a measurement speed of 1 million points per second. The vehicle equipped with the MMS device obtained the point clouds of the slope at a distance of approximately $12 \mathrm{~m}$ from the target slope while traveling at a speed of $50 \mathrm{~km} / \mathrm{h}$ in the overtaking lane (Fig.8). The first- and second-day point clouds were acquired on January 13, 2018, and May 28, 2018, respectively. For GNSS surveying, we used the position dilution of precision (PDOP) for quality indication. Conventionally, a time zone with a PDOP of less than three is considered as a good condition [10]; therefore, MMS measurements were carried out during this time zone. The acquired point cloud was corrected using the target of the reference points for adjustment.

For TS measurement, we conducted two hours of static surveying at two locations near both ends of the slope (GNSS points 1 and 2) using the GNSS surveying equipment (Fig.9). Using the GNSS points, the TS location was observed from the backward intersection. For error reduction, a radial triangulation survey and a countermeasure survey were used. The observed point was set as the TS installation point and the slope was measured at this point.

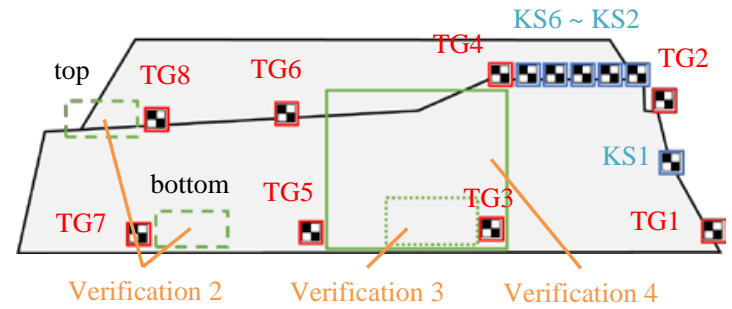

Fig.7 View of the target slope. TG are reference points for adjustment, KS are verification points. Verification (i) verified the targets. Verifications (ii), (iii), and (iv) verified the ranges

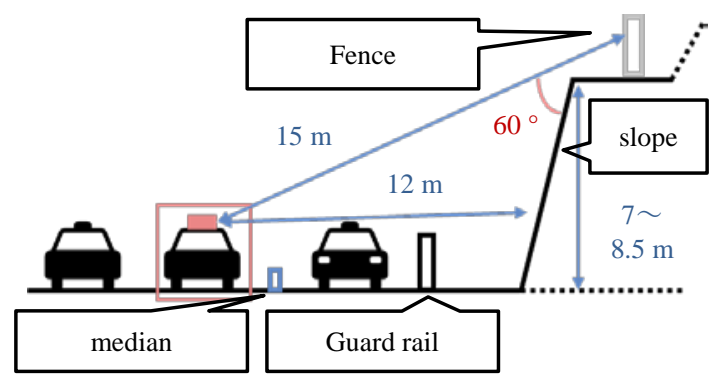

Fig.8 Various distances between the MMS vehicle and the target slope during the measurement

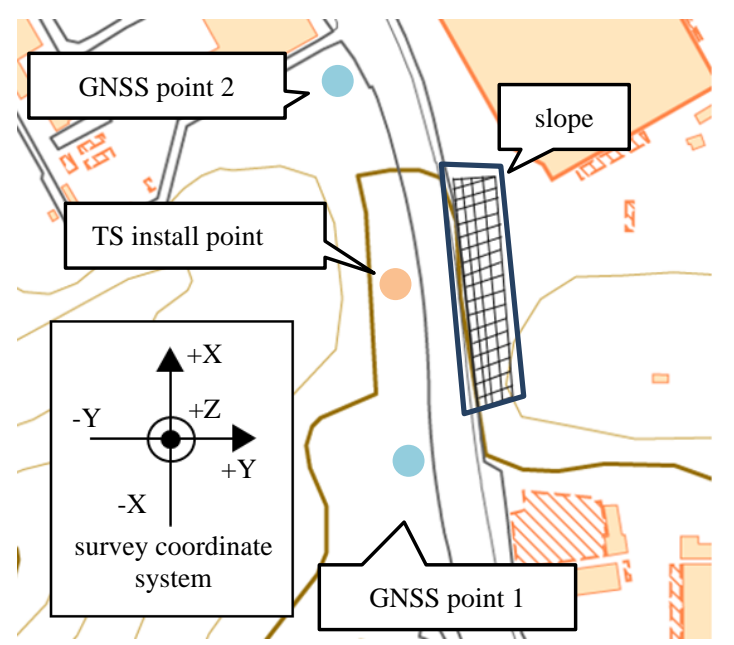

Fig.9 Relationship between the slope, GNSS points, TS installation points, and the survey coordinate system

\section{FIRST VERIFICATION: ACCURACY OF THE MMS POINT CLOUD ACQUISITION}

\subsection{Overview}

For the first verification method, we compared the center coordinates of the target acquired by the MMS and TS measurements; the obtained result was denoted as the accuracy of the point cloud acquired using the MMS measurement. Each point 
cloud was represented by $\mathrm{X}, \mathrm{Y}$, and $\mathrm{Z}$ in the plane rectangular coordinate system VI system [11].

The center coordinates of the six KS target points included in the MMS acquired point cloud were obtained using CloudCompare [12] and compared with the six KS targets obtained from the TS measurement. The mechanical accuracy of the TS measurement for the observational distances of $30 \mathrm{~m}$ to $100 \mathrm{~m}$ was $\pm 2.2 \mathrm{~mm}$. Since the differences between two separate days was obtained due to the TS mechanical accuracy, the TS coordinates of each day were used as the true values.

The KS center coordinates of the MMS and TS measurements were compared using Eq. (1) and (2). The point cloud acquisition accuracy of the MMS measurement was used in Eq. (3).

$$
\begin{aligned}
& D I S=O B S-T V \\
& \overline{D I S}=\frac{\left(\sum D I S\right)}{n} \\
& R M S E=\sqrt{\frac{\left(\sum D I S^{2}\right)}{n}}
\end{aligned}
$$

Herein, DIS , OBS , TV , $\overline{D I S}, n, R M S E$, and RMSE represent the difference, observed value, mean value of the difference, number of verification points, root mean square error, and the error of the MMS survey, respectively. DIS is the difference between the coordinates acquired by the MMS and TS measurements. RMSE is the variability of the point cloud in the $\mathrm{X}, \mathrm{Y}$, and $\mathrm{Z}$ directions where the difference is the most probable value.

\subsection{Results and Discussions}

This section explains the results of the errors in MMS measurements acquired in this verification.

The results are shown in Table 1 . The difference between the two GNSS reference points and the TS installation point was $0.000 \mathrm{~m}$ in two separate days. The difference of the six verification points was about $2 \mathrm{~mm}$, which was about the same as the TS mechanical accuracy. No deformation was assumed to have occurred in the two separate days, and the accuracy was verified.

The results are shown in Table 2, 3. These tables show the difference of the point cloud acquired by MMS and TS measurements. Table 2 is the result before adjustment using TG, Table 3 is the result after.

Table 1 Coordinates of KS points acquired by TS measurement, TG points acquired by GNSS measurement, TS installation points, and their differences. The unit is $\mathrm{m}$.

\begin{tabular}{|c|c|c|r|r|r|r|r|r|r|}
\hline & \multicolumn{3}{|c|}{ TS (first measurement) } & \multicolumn{2}{c|}{ TS (second measurement) } & \multicolumn{3}{c|}{ Difference (second - first) } \\
\cline { 2 - 10 } & $\mathrm{X}$ & $\mathrm{Y}$ & $\mathrm{Z}$ & $\mathrm{X}$ & $\mathrm{Y}$ & $\mathrm{Z}$ & $\Delta \mathrm{X}$ & $\Delta \mathrm{Y}$ & $\Delta \mathrm{Z}$ \\
\hline KS1 & -75570.418 & -81758.332 & 52.236 & -75570.419 & -81758.333 & 52.234 & -0.001 & -0.001 & -0.002 \\
\hline KS2 & -75565.143 & -81760.042 & 57.327 & -75565.144 & -81760.043 & 57.325 & -0.001 & -0.001 & -0.002 \\
\hline KS3 & -75562.682 & -81760.481 & 57.500 & -75562.684 & -81760.480 & 57.500 & -0.002 & 0.001 & 0.000 \\
\hline KS4 & -75560.092 & -81760.852 & 57.429 & -75560.094 & -81760.851 & 57.429 & -0.002 & 0.001 & 0.000 \\
\hline KS5 & -75557.592 & -81761.159 & 57.314 & -75557.594 & -81761.158 & 57.315 & -0.002 & 0.001 & 0.001 \\
\hline KS6 & -75555.039 & -81761.428 & 56.932 & -75555.040 & -81761.428 & 56.931 & -0.001 & 0.000 & -0.001 \\
\hline GNSS 1 & -75615.386 & -81773.663 & 44.120 & -75615.386 & -81773.663 & 44.120 & 0.000 & 0.000 & 0.000 \\
\hline GNSS 2 & -75460.249 & -81797.344 & 48.685 & -75460.249 & -81797.344 & 48.685 & 0.000 & 0.000 & 0.000 \\
\hline TS point & -75561.820 & -81784.723 & 46.465 & -75561.820 & -81784.723 & 46.465 & 0.000 & 0.000 & 0.000 \\
\hline
\end{tabular}

Table 2 Coordinates of KS points acquired by MMS measurement before adjusting the measurements and KS points acquired by TS measurement. The unit is $\mathrm{m}$.

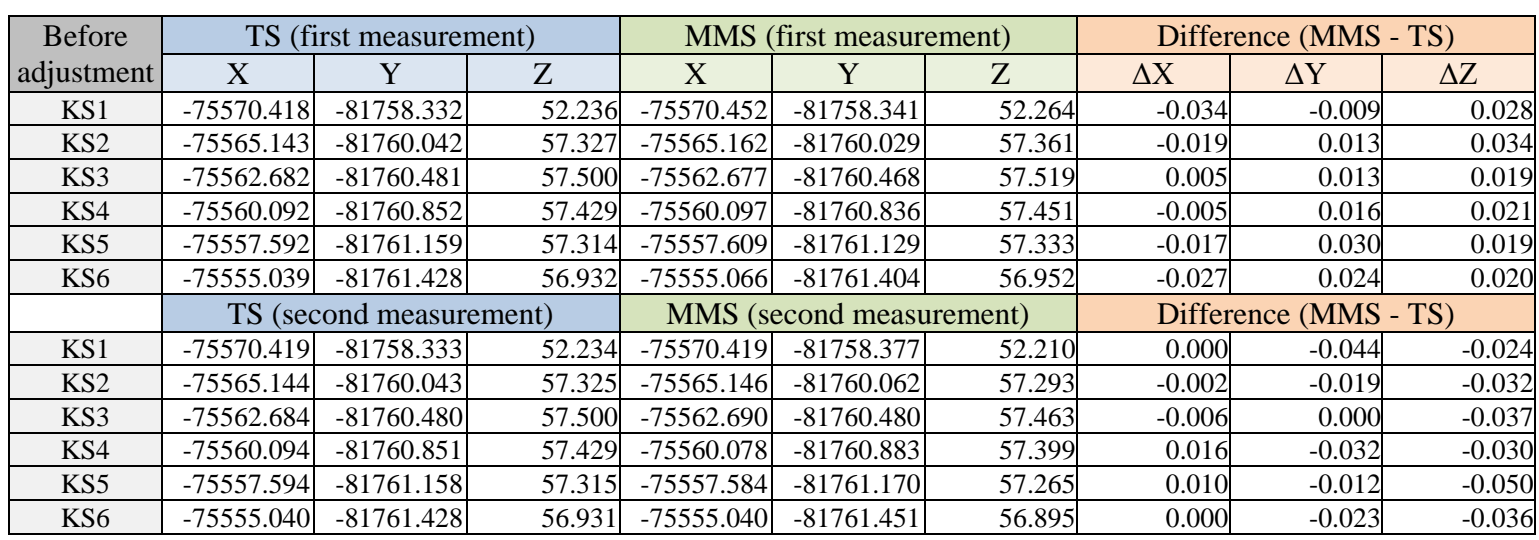


Table 4 summarizes the results of Table 2 and Table 3. The difference before and after the adjustment are separated, and the differences between the ground and the horizontal direction are shown as $\triangle X Y$, and the differences between the ground and the elevation direction are shown as $\Delta \mathrm{Z}$. The results obtained in Table 2 and Table 3 are summarized in terms of mean value, maximum absolute error and RMS error. Table 4 shows that the error of MMS measurement is $0.010 \mathrm{~m}$ for $\Delta \mathrm{XY}$ and $0.006 \mathrm{~m}$ for $\Delta \mathrm{Z}$ before adjustment, and $0.011 \mathrm{~m}$ for $\Delta X Y$ and $0.014 \mathrm{~m}$ for $\Delta \mathrm{Z}$ after adjustment. Comparing the mean values before and after adjustment, it can be seen that the values after adjustment are smaller. This result indicates that the TG adjustment reduces the systematic error value of the point cloud acquired MMS, in which all points have errors in a certain direction.

The accuracy of the MMS measurement is $11 \mathrm{~mm}$ in the $\mathrm{XY}$ direction and $14 \mathrm{~mm}$ in the $\mathrm{Z}$ direction.

\section{SECOND VERIFICATION: ACCURACY OF THE MMS POINT CLOUD REPRODUCTION}

\subsection{Overview}

To verify the accuracy of reproducing the deformations of the MMS acquired point cloud, we placed specimens that simulated two types of cracks and six types of bulges at the top and bottom of the slope. These cracks and bulges were represented by plates that simulated horizontal and vertical cracks, and plates of different thicknesses, respectively (Fig.10). We used cracks with a gap of 5, 10, 20, 30, 50 , and $100 \mathrm{~mm}$, respectively and bulges with a thicknesses of 2, 5, 10, 20,30, and $50 \mathrm{~mm}$, respectively. We checked the gaps and the thicknesses of each deformation to determine whether they could be identified in the MMS acquired point cloud. To check their reproducibility the cracks were placed at the top and bottom of the slope, respectively.

\subsection{Results and Discussions}

Fig.11 displays the point cloud of the specimen placed at the bottom of the slope. The point cloud had 818 points on TG7 of $0.7 \mathrm{~m}$ per side near the specimen; the point cloud density was 1670 points $/ \mathrm{m}^{2}$ at the bottom of the slope. In the lower part of the slope, we identified cracks with approximately $20 \mathrm{~mm}$ length in both the horizontal and vertical directions. The depth of the crack was indicated only when the width of the crack was more than $50 \mathrm{~mm}$ for horizontal cracks and $30 \mathrm{~mm}$ for vertical cracks. In the case of deformations, only bulges with a thickness of $10 \mathrm{~mm}$ or more were identified.

Fig.12 displays the point cloud of the specimen placed at the top of the slope. The point cloud had 544 points on TG8 of $0.7 \mathrm{~m}$ per side near the specimen; the point cloud density was 1088 points $/ \mathrm{m}^{2}$ at the top of the slope. In the upper

Table 3 Coordinates of KS points acquired by MMS measurement after adjusting the measurements and KS points acquired by TS measurement. The unit is $\mathrm{m}$.

\begin{tabular}{|c|c|c|r|r|r|r|r|r|r|}
\hline \multirow{2}{*}{$\begin{array}{c}\text { After } \\
\text { adjustment }\end{array}$} & \multicolumn{2}{|c|}{ TS (first measurement) } & \multicolumn{2}{c|}{ MMS (first measurement) } & \multicolumn{2}{c|}{ Difference (MMS - TS) } \\
\cline { 2 - 9 } & $\mathrm{X}$ & $\mathrm{Y}$ & $\mathrm{Z}$ & $\mathrm{X}$ & $\mathrm{Y}$ & $\mathrm{Z}$ & $\Delta \mathrm{X}$ & \multicolumn{1}{c|}{$\Delta \mathrm{Y}$} & $\Delta \mathrm{Z}$ \\
\hline KS1 & -75570.418 & -81758.332 & 52.236 & -75570.420 & -81758.326 & 52.217 & -0.002 & 0.006 & -0.019 \\
\hline KS2 & -75565.143 & -81760.042 & 57.327 & -75565.143 & -81760.030 & 57.314 & 0.000 & 0.012 & -0.014 \\
\hline KS3 & -75562.682 & -81760.481 & 57.500 & -75562.678 & -81760.465 & 57.477 & 0.004 & 0.016 & -0.023 \\
\hline KS4 & -75560.092 & -81760.852 & 57.429 & -75560.083 & -81760.849 & 57.411 & 0.009 & 0.004 & -0.018 \\
\hline KS5 & -75557.592 & -81761.159 & 57.314 & -75557.628 & -81761.149 & 57.326 & -0.036 & 0.010 & 0.011 \\
\hline KS6 & -75555.039 & -81761.428 & 56.932 & -75555.049 & -81761.422 & 56.935 & -0.010 & 0.006 & 0.003 \\
\hline \multicolumn{6}{|c|}{ TS (second measurement) } & \multicolumn{2}{|c|}{ MMS (second measurement) } & Difference (MMS - TS) \\
\hline KS1 & -75570.419 & -81758.333 & 52.234 & -75570.370 & -81758.315 & 52.214 & 0.049 & 0.018 & -0.020 \\
\hline KS2 & -75565.144 & -81760.043 & 57.325 & -75565.125 & -81760.038 & 57.295 & 0.019 & 0.005 & -0.030 \\
\hline KS3 & -75562.684 & -81760.480 & 57.500 & -75562.674 & -81760.490 & 57.514 & 0.010 & -0.010 & 0.014 \\
\hline KS4 & -75560.094 & -81760.851 & 57.429 & -75560.054 & -81760.875 & 57.429 & 0.040 & -0.024 & 0.000 \\
\hline KS5 & -75557.594 & -81761.158 & 57.315 & -75557.620 & -81761.156 & 57.325 & -0.026 & 0.002 & 0.010 \\
\hline KS6 & -75555.040 & -81761.428 & 56.931 & -75555.068 & -81761.400 & 56.926 & -0.028 & 0.029 & -0.005 \\
\hline
\end{tabular}

Table 4 Table summarizing the absolute calibration between the MMS and TS measurements (MMS-TS)

\begin{tabular}{|c|c|c|c|c|}
\hline \multirow{2}{*}{ Summary } & \multicolumn{2}{|c|}{ Before adjustment } & \multicolumn{2}{c|}{ After adjustment } \\
\cline { 2 - 5 } & $\Delta \mathrm{XY}$ & $\Delta \mathrm{Z}$ & \multicolumn{2}{c|}{$\Delta \mathrm{XY}$} \\
\hline Mean error & 0.027 & 0.023 & 0.016 & 0.010 \\
\hline Maximum absolute error & 0.036 & 0.034 & 0.037 & 0.023 \\
\hline RMS Error & 0.010 & 0.006 & 0.011 & 0.014 \\
\hline
\end{tabular}




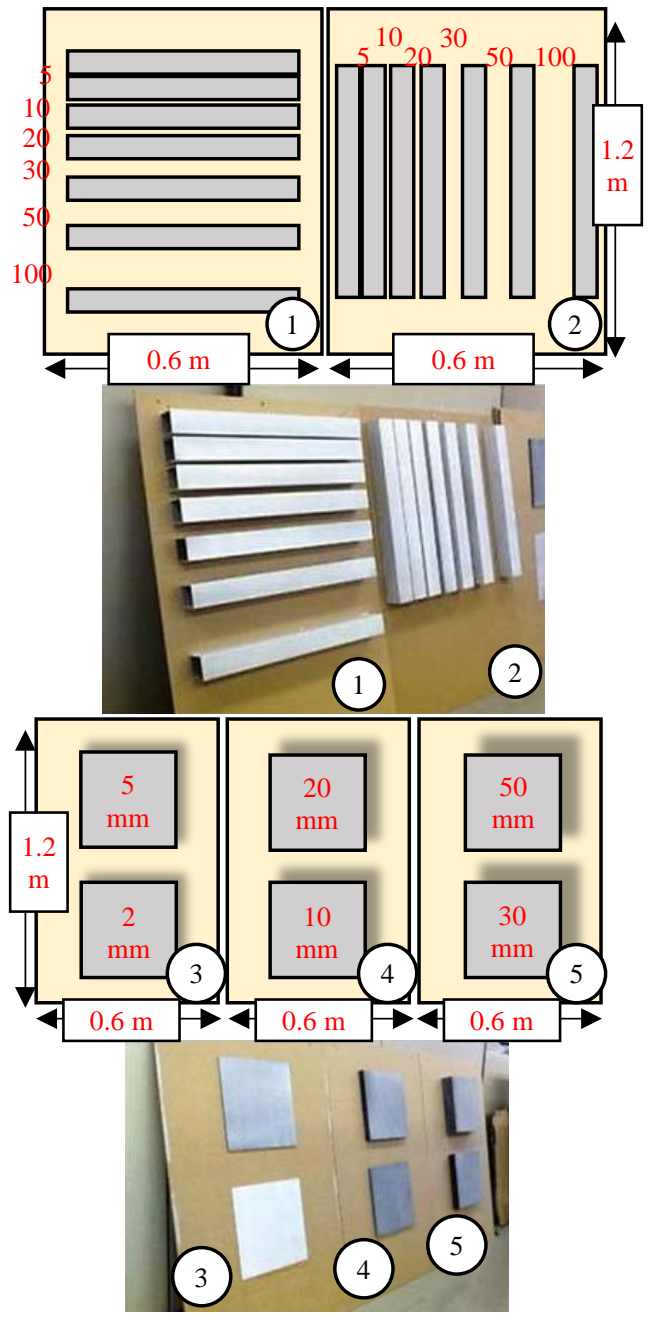

Fig.10 Specimens of cracks and bulges placed on the slope

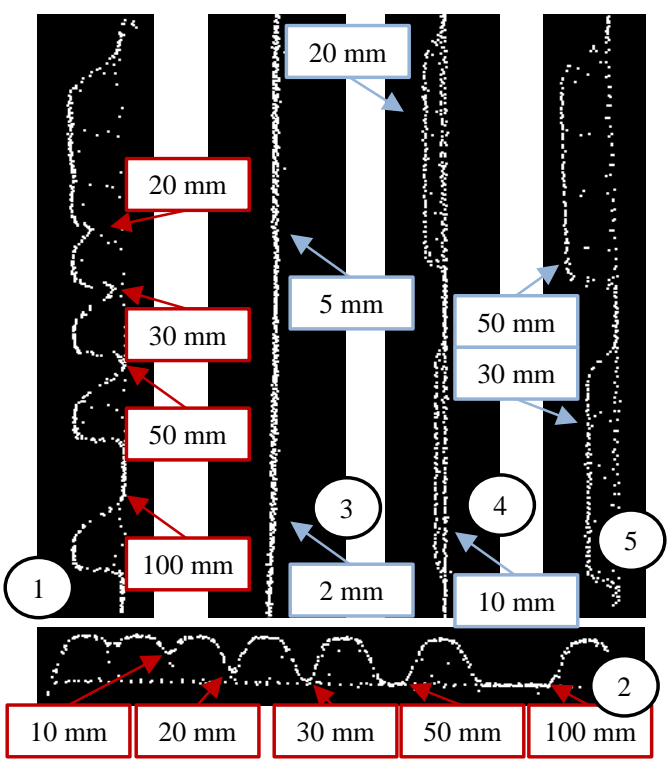

Fig.11 Point cloud of the specimen placed at the bottom of the slope

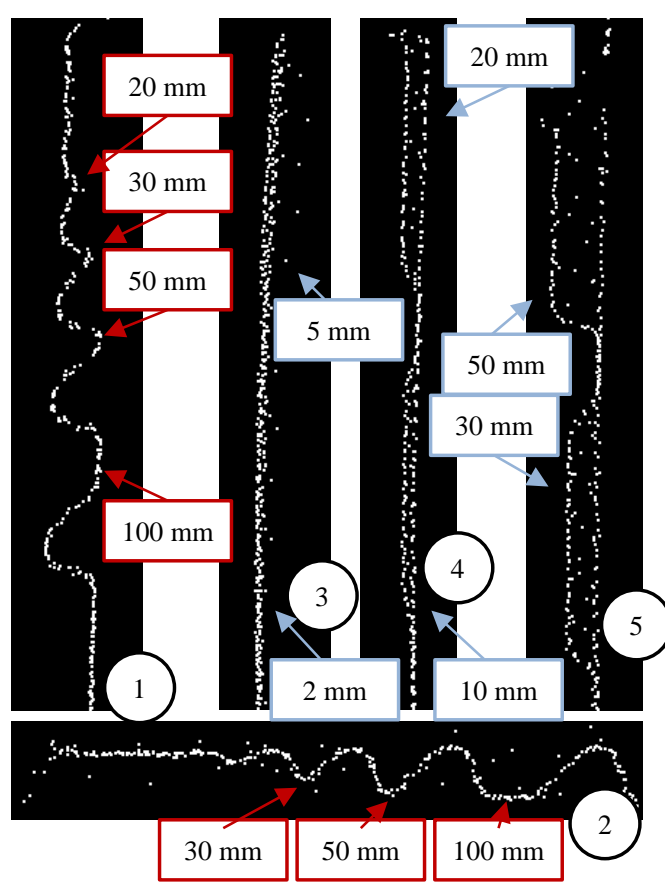

Fig.12 Point cloud of the specimen placed at top of the slope

part of the slope, we identified cracks were that were approximately $30 \mathrm{~mm}$ in both the horizontal and vertical directions. The depth of the crack was indicated only when the width of the crack was more than $50 \mathrm{~mm}$ both horizontally and vertically. In the case of deformations, only bulges with a thickness of $10 \mathrm{~mm}$ or more were identified.

\section{THIRD VERIFICATION: SLOPE DEFORMATION EXTRACTION USING THE ICP ALGORITHM}

To verify the extraction of slope deformations using the ICP algorithm, we used filtering to remove unnecessary points in the point cloud. Thereafter, we verified whether the results of the analysis altered depending on the presence or absence of filtering.

\subsection{Overview}

To extract the deformation from the slope, we obtained its point clouds using the MMS measurements on two separate days. Thereafter, the point cloud of the four slope frames in the center of the slope was cut out and used for verification.

To verify the extracted deformations, we denoted the targets on the four slope frames as A, B, $\mathrm{C}$, and D. For the second measurement, we set up simulated deformations and checked for changes by comparing the point clouds of the two separate days (Fig.13). The simulated deformations of each frame 
and the expected deformations obtained from the analysis are described as follows. In slope frame A of the second measurement, no simulated deformation was placed, and no change was expected to be observed on the two separate days. In slope frame $B$ of the second measurement, the surface was scraped off by approximately $40 \mathrm{~mm}$ near the center of the slope frame B; this change was expected to cause a change in the back of the slope. For the slope frames $\mathrm{C}$ and $\mathrm{D}$ of the second measurement, square sheets of $0.5 \mathrm{~m}$ per side and respective thicknesses of $10 \mathrm{~mm}$ and $20 \mathrm{~mm}$ were placed on both frames and a bulge was simulated. This simulated bulge deformation was expected to obtain quantifiable changes in the front of the slope. After conducting the analysis on all four slope frames, we performed filtering on the slope and conducted the analysis again. Thereafter, the results of the both analyses were compared. In the postfiltering analysis, the vegetation on the slope and the noise that may have been generated while measuring the point cloud of each slope frame. The mesh size for the analysis was set to $0.1 \mathrm{~m}$.

\subsection{Results and Discussions}

Fig.14 displays the magnitude of change represented by a heat map (wherein the closer the color blue is to red, the larger is the amount of change); the value to the right of each analysis result is the amount of change (unit: $\mathrm{m}$ ). The maximum value in these frames is obtained when the change is the largest in each frame. The figure on the left is a vector of the analysis results for the two separate days, separated by a $0.4 \mathrm{~m}$ section.

The results of the analysis were compared for each frame. In frame A, as expected, the extraction of the deformation could not be confirmed. In frame $\mathrm{B}$, where the surface was scraped off, deformation due to filtering was clearly observed.

In the cross section before filtering, deformation was observed in the area where icicles were found; in addition, we confirmed a deformation that changed in the direction of the back of the slope where the slope was cut. In frames $C$ and $D$, due to filtering, the changes in the slopes with $10 \mathrm{~mm}$ and $20 \mathrm{~mm}$ thick sheets were confirmed.

Based on these results, we confirmed that the laser point cloud acquired by the proposed MMS system could be used to extract deformations by applying the ICP algorithm. In addition, we confirmed that filtering the point cloud to extract only the necessary parts could clarify the deformation.
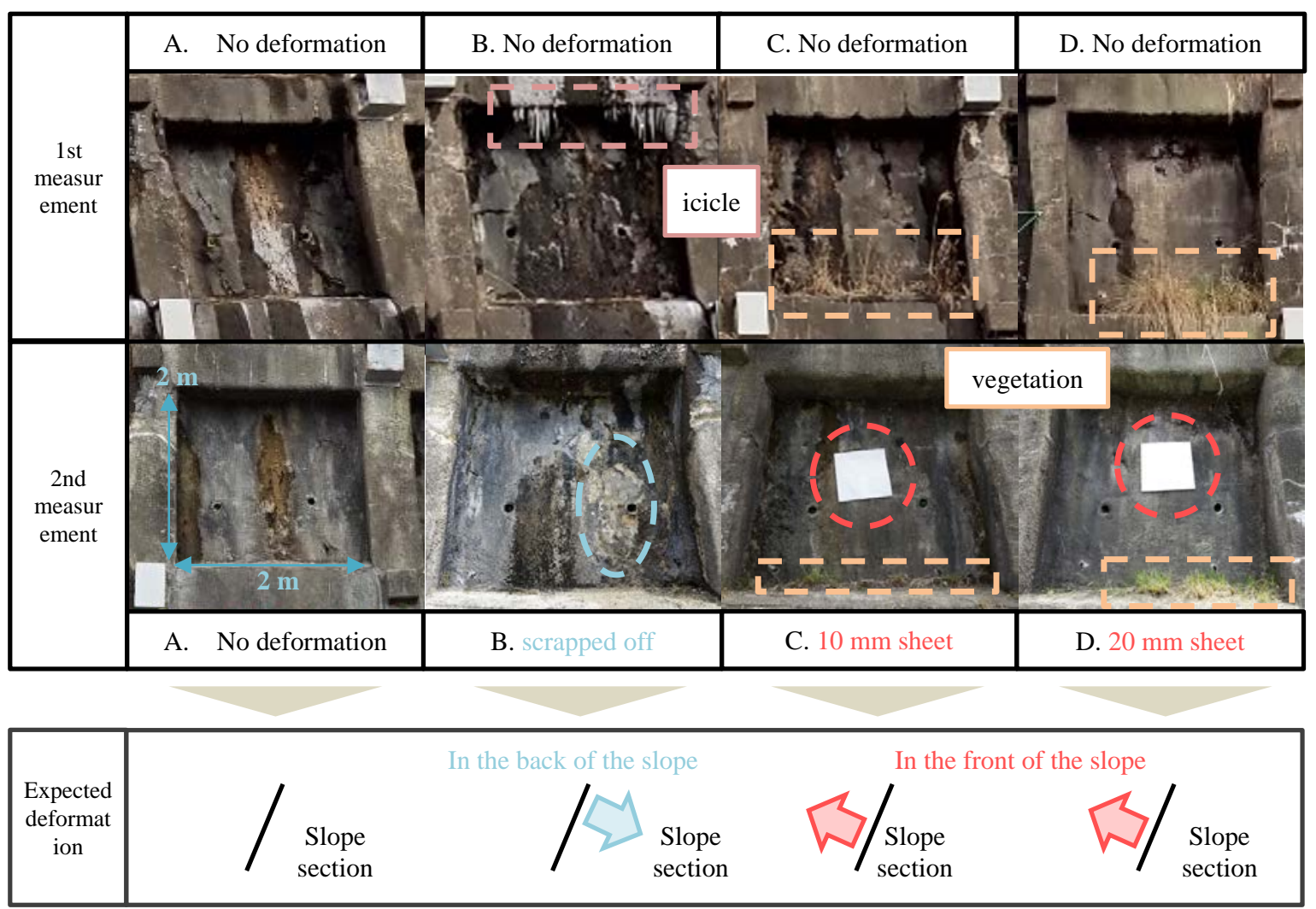

Fig.13 Slope with deformed specimens and the expected deformations for each slope 

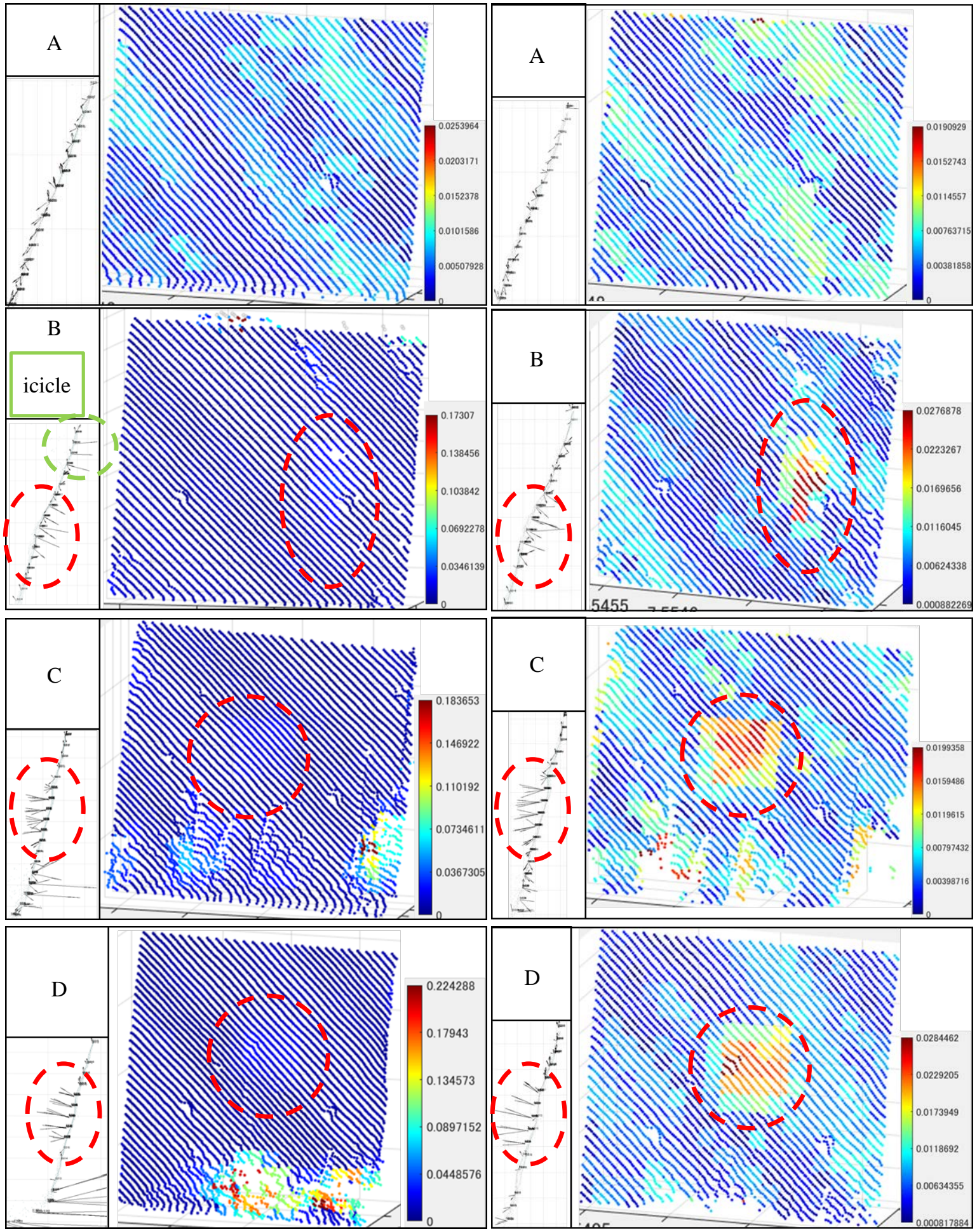

Fig.14 Analysis results of the slope frames before and after filtering (left: before filtering, right: after filtering) The larger the change, the closer it is to red, and the smaller the change, the closer it is to blue

\section{FOURTH VERIFICATION: SLOPE DEFORMATION EXTRACTION FROM A WIDE AREA}

\subsection{Overview}

The target for this verification was the slope, including the area where the simulated deformations were installed at the bottom in the second measurement of Section 5 (Fig.15). We verified whether the installed simulated deformations could be extracted by comparing the 
two point clouds of this slope obtained on the two separate days. For this verification, the point cloud of the slope was not filtered for vegetation and was cut out along the shape of the slope. To shorten the analysis time, for both measurements, the number of point clouds on the slope surface were reduced to half by using the random sampling function in CloudCompare, as displayed in Table 5. The mesh size for the analysis was set to $0.3 \mathrm{~m}$, and the maximum and minimum values of the deformation display were set to $0.05 \mathrm{~m}$ and $-0.05 \mathrm{~m}$, respectively.

\subsection{Results and Discussions}

The results of the analysis wherein the simulated deformations described in Section 5 were extracted are displayed in Fig.16. As per the figure, the area where the sheet was installed changed to the front of the slope, and the area where the scraped-off was removed changed to the back of the slope.

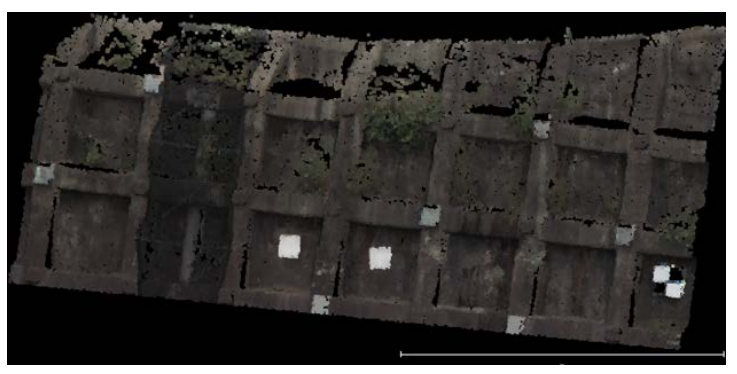

Fig.15 Point cloud of the second period of the target wide area slope
Table 5 Number of point clouds for two measurements for the slope point clouds.

\begin{tabular}{|c|c|c|}
\hline & \multicolumn{2}{|c|}{ Random sampling } \\
\hline $\begin{array}{c}\text { The number } \\
\text { of point cloud }\end{array}$ & Before & After \\
\hline $\begin{array}{c}\text { 1st } \\
\text { measurement }\end{array}$ & 159468 & 79734 \\
\hline $\begin{array}{c}\text { 2nd } \\
\text { measurement }\end{array}$ & 151372 & 75686 \\
\hline
\end{tabular}

\section{CONCLUSION}

In this study, considering the possibility of increased landslides on slopes, we extracted the deformation of the slope on two separate days using the laser point cloud acquired by the vehiclemounted sensing system (MMS) and the ICP algorithm. Thereafter, four performance parameters related to MMS and ICP were verified to determine whether the proposed method could aid slope inspection as a screening method.

First, the accuracy of the point cloud obtained using the MMS measurement was evaluated wherein the accuracy of the MMS measurement after adjustment was expressed in terms of RMS error. As per the results, the error was less than 11 $\mathrm{mm}$ in the horizontal direction $(\Delta X Y)$ and less than $14 \mathrm{~mm}$ in the elevation direction $(\Delta \mathrm{Z})$.

Second, we used specimens that simulated a crack or bulge deformation to verify the accuracy of

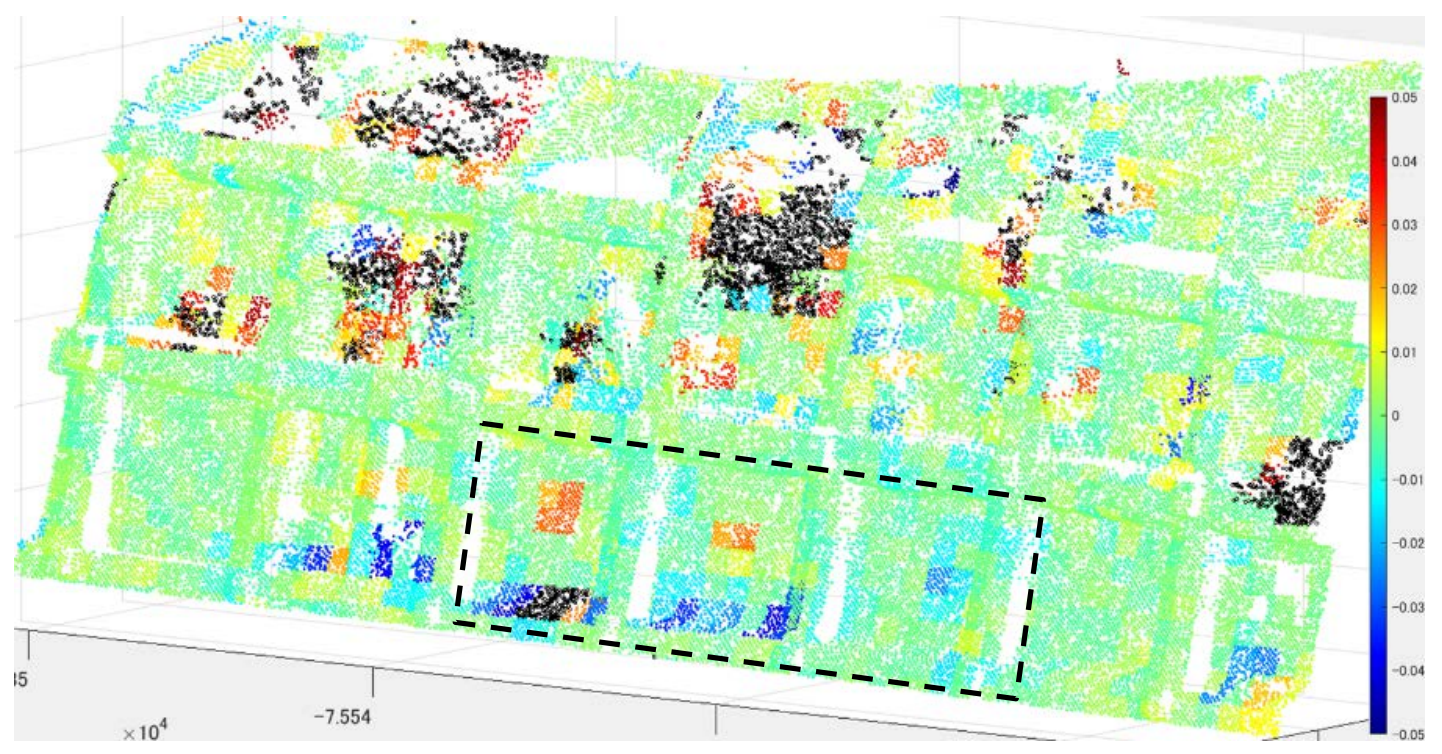

Fig.16 Analysis results. The black dotted line indicates the area where the simulated deformation was placed, as presented in Section 5. The black area indicates where the deformation was larger than $\pm 0.05 \mathrm{~m}$. 
the MMS point cloud with respect to reproducing these deformations. Based on the results, we detected and reproduced crack-like deformations at the bottom of the slope with a thickness of approximately $20 \mathrm{~mm}$ and bulge-like deformations with a thickness of more than $10 \mathrm{~mm}$.

For the third performance parameter, using simulated deformations such as scraped-off and bulge, we compared the slope frames obtained on the two separate days to verify whether these changes could be extracted by using the ICP method. As per the results, the ICP algorithm was able to extract deformations in front of the slope with a thickness of $10 \mathrm{~mm}$ and at the back of the slope. In addition, the algorithm enhanced the changes by removing point clouds such as vegetation and noise from the measurement.

Lastly, we verified whether the installed simulated deformations used in the third verification could be extracted in a wide area. As per the results, a $20 \mathrm{~mm}$ thick sheet was extracted as deformation.

Considering the obtained findings, we believe that the proposed method can play an important role in slope inspection. In addition, since this method uses quantitative data such as point clouds, it offers effective maintenance management by accumulating and managing data over long time periods; the data can be accumulated by cutting out the slope at a certain coordinate and giving it a number. Further, accumulating the changes over time would assist in determining the timing of repairs and inspections. With respect to the future prospect of this research, we want to improve the accuracy of the quantification of deformation since it will substantially contribute to future maintenance management through precise understanding of the progress of deformation.

\section{ACKNOWLEDGMENTS}

We would like to thank Kokusai Kogyo Co., Ltd. and Ministry of land, Infrastructure, Transport and Tourism Kinki Regional Development Bureau for their cooperation with this research.

\section{REFERENCES}

[1] Retrieved from https://www.data.jma.go.jp/cpdinfo/monitor/20 20/pdf/ccmr2020_all.pdf [Accessed: 2021-06$12]$

[2] Road Bureau, Ministry of Land, Infrastructure, Transport and Tourism, Inspection Guidelines for Road Earthwork Structures, 2017, pp. 7-12.

[3] Retrieved from https://psgsv2.gsi.go.jp/koukyou/public/tls/inde x.html [Accessed: 2021-06-12]

[4] Tachibana K., Mano K., Shimamura H. and Nishiyama S., The Application of Mobile Mapping System to River Embankment Measurement, Journal of the Japan Society of Photogrammetry and Remote Sensing, Vol. 54, Issue 4, 2015, pp. 166-177.

[5] Suehisa H., Measurement of Road Facilities Using High Standard Measurement Vehicle, Journal of the Japan Society of Photogrammetry and Remote Sensing, Vol. 47, Issue 5, 2008, pp. 28-31.

[6] Mano K., Izeki S., Morita S., Tachibana K. and Nishiyama T., Height Measurement of River Bank Crown by MMS, Journal of the Japan Society of Proceedings of JSCE F3, Vol.73, No.2, 2017, pp. I_53-I_64.

[7] Oban S. and Korodai M., Measurement of Civil Engineering Structures using MMS, Journal of the Japan Society of Photogrammetry and Remote Sensing, Vol.55, No.2, 2016, pp. 86-87.

[8] Paul J.B. and Neil D.M., A Method for Registration of 3-D Shapes, IEEE Transactions on Pattern Analysis and Machine Intelligence, Vol.14, No.2, 1992, pp. 239-256.

[9] Retrieved from https://leica-geosystems.com//media/files/leicageosystems/products/brochure s/leica_pegasustwo_bro.ashx?la=jajp\&hash=9360271D56FDB7ACEBEFC893D6 4B092E [Accessed: 2021-11-16]

[10] Koziroi R., Easy-to-understand GPS surveying, Omu Publisher, 2010, p.41.

[11] Retrieved from https://www.gsi.go.jp/LAW/heimencho.html [Accessed: 2021-07-15]

[12] Retrieved from http://www.danielgm.net/cc/ [Accessed: 2021-07-06]

Copyright (C) Int. J. of GEOMATE All rights reserved, including making copies unless permission is obtained from the copyright proprietors. 